Though I am reluctant to appear to advertise dealers, for the convenience of readers I think I am warranted in stating where the materials mentioned in this article may be purchased. The shade cloths may be purchased from the Remien and Kuhnert Co., 61 W. Grand Ave., Chicago. In September, 1916, I was given a price of 38 cents per yard for "Peerless Cambric Ivory White" shade cloth, 48 inches wide in entire rolls. The price was 44 cents per yard in small quantities. The White Holland shade cloth was slightly cheaper. Dr. G. R. LaRue, of the University of Michigan, has informed me recently that "Linaura Chart Cloth" sold by the Williams, Brown \& Earle Co., Philadelphia, is very satisfactory.

For labelling, we are using a so-called "Sign and Price Marker" set, No. 48 in catalogue No. 28 of Meyer and Wenthe, 108 N. Dearborn St., Chicago. The catalogue price is $\$ 5.00$ for the complete set. It is adapted to charts to be used in large lecture rooms. Set No. 6 at $\$ 4.00$ and set No. 4 at $\$ 2.50$ are recommended for smaller rooms. The round writing pens can probably be bought at many art and drafting instrument stores. Mine were obtained of A. H. Abbot and Co., 119 N. Wabash Ave., Chicago, $\frac{1}{4}$ gross for 25 cents.

ANATOMICAL LABORATORIES,

VANDERBILT UnIVERSITY MEdicaL School

\section{THE ELEMENTARY TREATMENT OF FORCE}

The discussion by G. S. Fulcher in ScIence, XLIV., 747, 1916, concerning some of the errors and inconsistencies in our elementary texts regarding the questions of force and Newton's laws of motion, are most timely. No doubt many of us who are trying to build up in the minds of our beginning students a sound structure of physical ideas, and above all, are hoping through physics to give them something of the scientific attitude, have almost despaired of finding a text which is free from the faults mentioned. To approach the ideal, a text should be brief in its statements but so explicit as to allow of but a single interpretation; it should not anticipate knowledge which obviously the beginning student does not possess, nor should it attempt to circumvent this deficiency by repeatedly referring the student to articles further along; it should, in fact, be written upon the premise that the only source of physical ideas which the average beginning student of physics has is his own experience.

In introducing force, therefore, all speculation and conjecture, made in the light of the author's own familiarity with the subject, is decidedly out of place, and can serve only to confuse the student. It should be presented to him primarily in terms of his immediate impressions, $i$. e., in terms of his muscular sense. Let us tell him first that "force" is the term applied to the equivalent of a push or a pull. The average student has pretty clear ideas as to what such an action can accomplish. It is then not difficult to represent Newton's first law as a test for the absence of a force, nor the second law as a test for its presence. After familiarity with these notions has been gained, we can further represent the second law as a quantitative test for force, and can show how we can experimentally establish the relation $f=m a$. This may then be regarded as a more exact definition of force, derived from our observations upon objects external to ourselves. In all of this discussion it is of greatest importance to emphasize by repetition the fact that whenever a force is exerted, two bodies are involved: $A$, the body acting, and $B$, the body acted upon. ${ }^{1}$ This is one of the outstanding

1 In his reply (ScIEnCE, XLV., 480, 1917) to A. H. Patterson (SCIENCE, XLV., 259, 1917), which was printed after the present paper had been submitted for publication, Dr. Fulcher has already emphasized this point.

In this connection, may I suggest that we discontinue the use of the phrase, "a force acts upon ...." which is so exceedingly common in our texts? It seems to me that the phrase attributes to force a property which it does not possess. Why not be unequivocal and say "a force is exerted upon ..."'? This latter way of stating the fact serves better than the former in keeping the above italicized principle before the student, in that it deprives the notion of force of that seeming independence which does not pertain to it. 
points which have been disregarded in our texts. The result has been that in a large number of cases the student did not see how the force was being exerted; he felt that there was an intangible something about force beyond the range of his ability to grasp. It is small wonder that rapidly increasing confusion results from this lack of self-confidence.

Another point can not be too strongly emphasized. No author of an elementary text seems as yet to have recognized the importance of distinguishing most carefully between forces of action and forces of reaction. It seems to me of prime importance, if the student's ideas about force are to be sharply outlined, to make this distinction. I would suggest that it lends facility to the treatment to use the terms " applied" force and "reactive" force for forces of action and of reaction, respectively. The following treatment may bear out the contention concerning the importance of recognizing both.

Let us first apply Newton's third law of motion to the case of a body $A$, acting upon a fixed body $B$. In this case the student readily understands that $B$ exerts a reactive force equal and opposite to the applied force exerted by $A$; for if this were not true, motion should ensue in accordance with the second law. He also readily sees that the reactive force arises and ceases simultaneously with the applied force. Often, however, when the third law is applied to a movable body $B$, and the student is told that the accelerated $B$ exerts a reactive force upon $A$, equal and opposite to the applied force exerted by $A$, he is dubious and asks: "If that is true, how can there be an acceleration? By the first law, $B$ should then remain at rest or in uniform motion." With the distinction between applied and reactive forces clearly drawn, this question can not arise. In this particular case we may point out that, to be sure, no acceleration of $B$ could occur if the force exerted by $A$ upon $B$ were opposed by another applied force, exerted upon $B$ in the opposite direction by a third body, $C$. However, since there is only the one applied force, that due to $A$, acceleration must ensue in accordance with Newton's second law; and, simultaneously with this acceleration, ergo, with the applied force, there arises the reactive force of $B$ upon $A$. We may, to present the case somewhat more tangibly, speak of the applied force as being exerted by that particular body which, so to speak, takes the initiative in the processes. It is well to point out, further, that the reactive force of $B$ upon $A$ arises regardless of whether the motion of $B$ is in the direction of the force exerted by $A$, or whether there is a finite angle between these two directions. If this angle is a right angle, the applied force causes $B$ to move in a circular path, without change in speed; the applied force is then called centripetal. The reactive force exerted by $B$ upon " $A$ under these conditions is called the centrifugal force, which disappears at exactly that instant which marks the disappearance of the applied force. Numerous examples to illustrate these statements will occur to the teacher.

If the student is familiar with the above principles, the problem of Atwood's machine becomes very simple. For here considering the moving system as a unit, the applied force of $A$ (the earth) upon $B$ (the system) is obviously $\left(m_{2}-m_{1}\right) g$; the reactive force of $B$ upon $A$ is $\left(m_{2}+m_{1}\right) a$. These, by the third law, are equal. The value of $a$, or of $g$, immediately follows from the equality.

I can not quite agree with Dr. Fulcher that the failing of our authors in treating reactive forces, especially centrifugal force, as they do, is because of "their forgetting that these forces are purely imaginary." Is it not rather attributable to their previous neglect in not having emphasized the difference between applied and reactive forces?

Just an illustration, in conclusion, to show that American writers do not alone fall under the criticism of giving insufficient thought to the presentation of some of the fundamental things. We find, for example, in a German text, widely used in the Realschulen, by an author reputed to have been one of the foremost teachers of physics, a treatment as follows: "Upon the liquid particle $A$ there are acting two forces, the force of gravity, ver- 
tically downward, and the centrifugal force, acting horizontally. . . . The resultant is found by the parallelogram law. Its direction must be normal to the surface of the liquid." Had this author, and our American authors, been careful to draw the distinction between applied and reactive forces, they could not easily have fallen into the error of combining an applied force with a reactive force and obtaining-what kind of a force? Whatever the kind, it can not be an applied force; for if it were, it should, according to the second law, produce an acceleration in its own direction. But such an acceleration, as pointed out by Dr. Fulcher, does not here exist.

There are many other fundamental questions in physics about the best method of presentation of which we are not agreed. Teachers of college physics should welcome the opportunity of discussing them, and by so doing, clearing up their own ideas about them. Perhaps, also a thoroughly satisfactory text might thereby be evolved.

\section{UNIVERSITY OF MiNNESOTA}

\section{PaUl E. Klopsteg}

\section{QUOTATIONS}

\section{ROYAL SOCIETY FELLOWSHIPS}

A QUESTION of more than ordinary interest and importance is involved in the opposition of a majority of the Fellows of the Royal Society to a proposal of its council to amend the statute of the society governing the election of fellows. On June 7 a special general meeting of the Royal Society was held, as the result of a petition to the council, to consider a proposal by the latter embodied last year in their report for 1916. It was to amend Statute XII. by empowering the council to recommend for election (a) privy councillors "whose election would assist the work of the society"; and (b) "men distinguished in the scientific or educational service of the state, or by their services to science and its applications." The opposition to this proposal, led by Sir David Bruce and Sir E. Ray Lankester, had been energetically whipped up among the unofficial fellows since last November, and there was an unusually large attendance at the meeting. The result was that a vote was taken, adverse by a considerable majority to the council. The following resolution was carried: "That this meeting is of opinion that the council will serve the best interests of the society by restoring Statute XII. to the form it had before the change made in it by the council on November 2, 1916, and by postponing further consideration of the statute relating to the election of fellows until after the termination of the war." The precise effect of the action thus taken by a majority of the fellows is for the moment rather uncertain, and the position is a somewhat embarrassing one for the president and council, who have thus suffered an apparent rebuff. According to the constitution of the Royal Society, the power of making and amending its statutes resides solely in the council, so that, strictly, the resolution is a brutum fulmen. On the other hand, the actual election of fellows rests with the society, and the council can only recommend candidates. So that the council is hardly likely to provoke an unseemly opposition to candidates it might recommend for election under the amended statute-even if it declines to stultify itself by "restoring" the status quo ante as suggested -by flying in the face of the adverse vote.

We understand that the president and council were, in fact, quite ready to meet the opposition raised within the society so far as concerns a postponement of any action on the amended statute till after the war. And in the comment we propose to make we can not, partly for that reason, express our entire disagreement with the opposition too strongly at this juncture. At the same time we think it desirable to say at once that we think the hostility of so many fellows to a proposal intended to increase the prestige and the value of the Royal Society distinctly regrettable. It was based, we are well aware-at any rate among some of the more eminent fellows who led the opposition-largely on suspicions of the introduction of state patronage into scientific research. But we have no doubt also that the influence of "vested interests" in the existing system of election to the coveted distinction of 